\title{
El Manejo de la Reserva Biológica Uyuca en el Contexto Nacional y Global del Sistema de Áreas Protegidas
}

\author{
José Manuel Mora ${ }^{1}$ y Lucía I. López ${ }^{2}$
}

\begin{abstract}
Resumen. La mayoría de las sociedades humanas desarrollaron relaciones de respeto e incluso de adoración con la naturaleza. La biodiversidad del planeta experimenta una fuerte crisis cuyos orígenes radican en el modo de relación establecido por la sociedad industrial con el mundo natural. Esta situación ha llamado la atención mundial y ha justificado la creación de áreas silvestres protegidas para mantener muestras representativas de la biodiversidad. Sin embargo, la conservación biológica y la ética ambiental no se pueden separar de la justicia social. Debido a ello, las áreas protegidas deben ser un componente vital de cualquier estrategia de conservación y a la vez servir de hospedero de otras necesidades sociales, culturales y económicas. Se reconoce que las áreas protegidas pueden ser fuentes de importantes beneficios económicos, por lo que pueden ser un aporte significativo a las economías locales. La Reserva Biológica Uyuca (RBU) es una pequeña área protegida creada en 1985 que ocupa la porción más alta del cerro Uyuca a $15 \mathrm{~km}$ al oriente de Tegucigalpa. Desde 1948, Zamorano ha trabajo en pro de la conservación de la biodiversidad de todo el cerro Uyuca. El manejo actual de esta reserva está a cargo del Departamento de Ambiente y Desarrollo (DAD) de la Escuela Agrícola Panamericana. La RBU ha sido establecida legalmente para limitar el alcance de la conversión de tierras dentro de su área delimitada. Hay 10 aldeas que se beneficien directamente del agua de la RBU, pero la presión y demanda está en aumento. Recientemente, el DAD elaboró un nuevo plan de manejo para la RBU que cumple todos los requisitos del Instituto Nacional de Conservación Forestal de Honduras. El nuevo plan provee los principios necesarios para el manejo adecuado de la RBU y permite la participación de la sociedad civil en la elaboración de este elemento clave según los lineamientos del sistema nacional de áreas protegidas.
\end{abstract}

Palabras clave: Biodiversidad, comanejo, ecosistemas, plan de manejo, Zamorano.

\section{Management of the Uyuca Biological Reserve in the National and Global Context of the Protected Areas System}

\begin{abstract}
The majority of human societies developed relations of respect and even of adoration with nature. The biodiversity of the planet is experiencing a strong crisis whose origins take root in relationship established by the industrial society with the natural world. This situation has called the world's attention and has justified the creation of wild protected areas (AP) in order to maintain representative samples of the biodiversity. Nevertheless, biological conservation and the environmental ethics cannot be separated from social justice. Due to this, the protected areas must be a vital component of any conservation strategy and simultaneously be used as inn-keeper of other social, cultural and economic needs. It is admitted that the AP can be a sources of important economic benefits so they can be a significant contribution to local economies. The Uyuca Biological Reserve (UBR) is a small protected area created in 1985 that occupies the highest portion of the Uyuca Mountain, $15 \mathrm{~km}$ East of Tegucigalpa. However, since 1948, Zamorano has worked in pro of the conservation of the biodiversity of the entire Uyuca Mountain. The current management of the UBR is a responsibility of the Department of Environment and Development (DAD) of the Escuela Agrícola Panamericana. The UBR has been established legally to limit the scope of the conversion of lands inside its delimited area. There are 10 villages that benefit directly of the water of the UBR but the pressure and demand for water is on the increase. Recently, the DAD elaborated a new management plan for the UBR that fulfills all the requirements of the National Institute of Forest Conservation of Honduras. This new plan provides the principles for an adequate management of the reserve but it also allowed the participation of the civil society in the elaboration of this key element following the guidelines of the national system of protected areas.
\end{abstract}

Key words: Biodiversity, co-management, ecosystems, management plan, Zamorano.

\footnotetext{
${ }^{1}$ Centro Zamorano de Biodiversidad, Departamento de Ambiente y Desarrollo, Escuela Agrícola Panamericana, Zamorano, Honduras, correo electrónico jmora@zamorano.edu

${ }^{2}$ Consultora Ambiental, ecología acuática y macroinvertebrados acuáticos, correo electrónico luciaisa2@ gmail.com
}

DOI: $10.5377 /$ ceiba.v52i1.976 


\section{Introducción}

La relación de la humanidad con las áreas naturales se remonta a los comienzos de nuestra historia evolutiva ya que ese era nuestro entorno inmediato. Las sociedades recolectoras-cazadoras debieron desarrollar una relación benigna con las plantas y los animales de las que ellos dependían (Dasmann 1981). A través de la historia, los gobiernos impusieron leyes para resguardar los recursos naturales de alguna forma y con diferentes objetivos. En las épocas medievales, la fauna silvestre de Gran Bretaña estaba bajo la corona y la nobleza. El derecho de cazar lo tenía el rey y se les concedía derecho de cazar a los aristócratas (Dasmann 1981). En 1564, las autoridades polacas establecieron una reserva donde la cacería era prohibida. Esta reserva representa uno de los primeros esfuerzos realizados en Europa para conservar las especies en áreas silvestres protegidas. Con la creación del primer parque nacional o área protegida moderna, el parque nacional Yellowstone en Estados Unidos en 1872, vino la aparición del concepto moderno de área protegida (Arguedas et al. 2004).

La relación de las sociedades con la naturaleza ha sido, en muchos de casos, de respeto e incluso de adoración. Varias culturas indígenas americanas conciben a los seres humanos como hijos y habitantes de la Tierra. En el altiplano suramericano, la Tierra o Pacha Mama, es para los quechuas una deidad, un ser vivo y dinámico a quien se le hacen ofrendas y se le respeta en las prácticas agrícolas y en las fechas de cosecha para mantener la fertilidad. Para los tukanos, que habitan los ecosistemas fluviales de la amazonia colombiana, el creador del universo es el Sol, quien creó solo un número finito de plantas y animales. Para habitar este universo de recursos limitados, los tukanos poseen numerosas regulaciones. Por ejemplo, los ríos pertenecen a los peces y toda tala en los bosques ribereños está prohibida (Primack et al. 2001).

La destrucción y la fragmentación del bosque, los cambios climáticos tales como el calentamiento global, el incremento en los incendios forestales sin control, la introducción de nuevas sustancias químicas al medio ambiente y la introducción de especies exóticas degradan la biodiversidad (Primack et al. 2001). En general, los orígenes de la crisis ambiental actual radican en el modo de relación establecido por la sociedad industrial con el mundo natural (Primack et al. 2001). Ahora se entiende que las amenazas a la biodiversidad son sinérgicas, es decir, varios factores independientes, tales como la lluvia ácida, la deforestación y la sobrecacería, se combinan en forma aditiva o incluso multiplicativa para agravar aún más la situación (Myers 1987). Este dilema ha llamado la atención mundial y ha justificado la creación de áreas silvestres protegidas con el fin de mantener muestras representativas de todos los ecosistemas terrestres y proteger la mayor cantidad de especies, poblaciones y genes (Primack et al. 2001). Asimismo, la mayoría de los países forman parte de convenciones o tratados internacionales y cuentan con estrategias y programas de conservación para salvaguardar la biodiversidad para las futuras generaciones (Meffe y Carroll 1997).

Las áreas protegidas son un componente vital de cualquier estrategia de conservación y a la vez sirve de hospedero de otras necesidades sociales, culturales y económicas. Las áreas protegidas cumplen funciones que pueden ser resumidas en tres grupos principales: servicios ambientales, beneficios sociales y económicos y el mantenimiento de los ecosistemas naturales y la biodiversidad (Carey et al. 2000). Aun así, la calidad de muchas áreas protegidas está en decline como resultado de un sinnúmero de amenazas y presiones. Un alto número adicional de áreas protegidas permanecen inseguras y su futuro a largo plazo es incierto (Carey et al. 2000).

Se supone que una vez que un área protegida ha sido identificada y declarada, sus valores serán preservados indefinidamente. Desafortunadamente, este no es el caso ya que la calidad del área protegida y la biodiversidad que contiene pueden sufrir de múltiples formas. Ese efecto negativo va desde la remoción de especies clave, a veces debido a cacería furtiva y varios tipos de daños ecológicos generales, hasta los casos extremos de destrucción casi total del área protegida. Incluso, en casos en que las áreas estén bien protegidas y relativamente intactas, pueden ser fuertemente afectadas debido al aislamiento y la fragmentación si las tierras aledañas tienen usos del suelo no sustentables. Las áreas protegidas enfrentan 
amenazas que van desde los impactos de los asentamientos humanos, la caza y pesca ilegal, hasta la contaminación del aire y el cambio climático (Carey et al. 2000). Las presiones aumentan porque muchas áreas protegidas en realidad no son áreas protegidas. Un alto número de áreas protegidas alrededor del mundo son parques de papel, lo que significa que el área fue declarada por un gobierno, pero nunca ha sido implementada. La declaración puede ayudar a proteger las áreas protegidas de algunas presiones, pero además se necesita una legislación adecuada, planes de manejo, personal, equipo, capacidad y por supuesto el apoyo y la cooperación de las comunidades aledañas (Carey et al. 2000).

La UICN (1994) describe un área protegida como una superficie de tierra o mar especialmente consagrada a la protección y el mantenimiento de la diversidad biológica, así como de los recursos naturales y los recursos culturales asociados y manejada a través de medios jurídicos u otros medios eficaces. Esta definición de área protegida hace referencia a los recursos culturales asociados, lo que refleja una concepción de la conservación que puede adaptar los intereses, valores, derechos y responsabilidades sociales, económicas y culturales de las comunidades locales que viven dentro o alrededor de las áreas protegidas (Beltrán 2001).

A través de los años, se ha desarrollado una diversidad de nombres para las áreas protegidas. En el 2012 hay más de 800 términos usados para describir las designaciones nacionales y sus regímenes de manejo. Dado esta diversidad de objetivos y sistemas de manejo, las categorías de manejo de áreas protegidas de la UICN (1994) juegan un papel crítico en los análisis regionales y globales. Dichas categorías proveen un lenguaje común y posibilita la comparación y el resumen de los objetivos de manejo para las áreas protegidas ya que posibilitan la interpretación de las definiciones nacionales de las áreas protegidas. El sistema de categorización de la UICN (Cuadro 1) es ampliamente aceptado y ha sido muy útil en obtener información más estandarizada y en proveer guía a los países cuando estos establecen y expanden su sistema de áreas protegidas. Es claro que el sistema aun tiene varias inconveniencias, pero ha proveído un acercamiento al entendimiento global de las áreas protegidas y su manejo.
Cuadro 1. Clasificación de las áreas protegidas propuesta por la UICN y que sirve como estándar internacional para manejo (Adaptado de diferentes publicaciones de la UICN).

\begin{tabular}{lll}
\hline Categoría & Objetivo principal \\
\hline Ia & $\begin{array}{l}\text { Reserva natural } \\
\text { estricta }\end{array}$ & Protección estricta \\
Ib & $\begin{array}{c}\text { Área silvestre } \\
\text { II }\end{array}$ & Parque nacional \\
III & Monumento natural & $\begin{array}{l}\text { Conservación de } \\
\text { ecosistemas y turismo } \\
\text { Conservación de } \\
\text { características naturales }\end{array}$ \\
IV $\quad$ Área de manejo de & $\begin{array}{l}\text { Conservación mediante } \\
\text { manejo activo }\end{array}$ \\
V $\quad \begin{array}{l}\text { Pábitat / especies } \\
\text { marino terrestre / }\end{array}$ & $\begin{array}{l}\text { Conservación y } \\
\text { recreación }\end{array}$ \\
VI & $\begin{array}{l}\text { Área protegida de } \\
\text { recursos manejados }\end{array}$ & $\begin{array}{l}\text { Uso sostenible de } \\
\text { ecosistemas naturales }\end{array}$ \\
\hline
\end{tabular}

El Parque Nacional es la categoría más conocida y más común y algunas veces erróneamente usado como el término genérico para todas las áreas protegidas. Los esquemas nacionales y la terminología de categorías de áreas protegidas pueden diferir, pero mayoritariamente siguen el modelo de la UICN. Asignar una de las categorías a un área protegida particular debe ser hecho después de un proceso científico y socialmente serio de definición de los objetivos del área protegida. Las categorías IV, V y VI se enfocan en la explotación controlada de los recursos, pero sin dejar de lado los compromisos del mantenimiento de la biodiversidad. En todo caso, cualquier categoría puede o no incluir asentamientos humanos, sistemas de transporte y uso comercial o de subsistencia mientras se acomoden dentro de un esquema de zonificación interna. Así, las actividades que son permisibles en cada categoría varían según los objetivos que tenga el área protegida (Cuadro 2).

La tendencia a promover el uso sustentable de la biodiversidad como un medio para protegerla es políticamente e intelectualmente conveniente. Sin embargo, este uso no siempre es compatible con la integridad de los sistemas vivos según el conocimiento que ha generado la biología. Ello implica que no todos los ecosistemas ni todos los demás componentes de la 
biodiversidad pueden estar abiertos al uso. Es necesaria una comprensión más amplia de la dinámica de los ecosistemas para evitar que las estrategias que promueven su uso sustentable lleven pérdidas sustanciales de biodiversidad. De igual manera, no se puede hablar de uso sustentable de todos los elementos de la biodiversidad ya que un sistema una vez intervenido tendrá pérdida de especies en mayor o menor grado (Redford y Richter 2002).

Cuadro 2. Matriz de los objetivos de manejo y las categorías de manejo (Cuadro 1) de las áreas protegidas de la UICN (Adaptado de UICN 1994).

\begin{tabular}{|c|c|c|c|c|c|c|c|}
\hline \multirow[b]{2}{*}{ Objetivos de manejo } & \multicolumn{7}{|c|}{ Categorías de manejo } \\
\hline & Ia & Ib & II & III & IV & $\mathbf{V}$ & $\overline{V I}$ \\
\hline Investigación científica & $1^{\S}$ & $3^{¥}$ & $2^{\mathbb{T}}$ & 2 & 2 & 2 & 3 \\
\hline Protección de la naturaleza & 2 & 1 & 2 & 3 & 3 & $\mathrm{na}^{\mathrm{p}}$ & 2 \\
\hline Preservación de la diversidad genética y de las especies & 1 & 2 & 1 & 1 & 1 & 2 & 1 \\
\hline Mantenimiento de los servicios ambientales & 2 & 1 & 1 & na & 1 & 2 & 1 \\
\hline Protección de características específicas naturales/culturales & na & na & 2 & 1 & 3 & 1 & 3 \\
\hline Turismo y recreación & na & 2 & 1 & 1 & 3 & 1 & 3 \\
\hline Educación & na & na & 2 & 2 & 2 & 2 & 3 \\
\hline Uso sostenible de los recursos de los ecosistemas naturales & na & 3 & 3 & na & 2 & 2 & 1 \\
\hline Mantenimiento de los atributos culturales/tradicionales & na & na & na & na & na & 1 & 2 \\
\hline
\end{tabular}

${ }^{\S} 1$ : Objetivo primario

II: Objetivo secundario

${ }^{\ddagger} 3$ : Objetivo potencialmente aplicable

'na: Objetivo no aplicable

\section{La Reserva Biológica Uyuca (RBU)}

La RBU es una pequeña área protegida que ocupa la porción más alta del cerro Uyuca. El manejo de esta reserva está a cargo del Departamento de Ambiente y Desarrollo (DAD) de la Escuela Agrícola Panamericana, Honduras. Zamorano ha tenido la responsabilidad del manejo de la reserva desde 1985 cuando, con un decreto de ley, el gobierno de la República de Honduras le delegó esta tarea. No obstante, desde 1948, Zamorano ha trabajado en pro de la conservación de la biodiversidad de todo el Cerro de Uyuca. Recientemente, el DAD elaboró un nuevo plan de manejo para la RBU. El plan de manejo cumple todos los requisitos del Instituto Nacional de Conservación Forestal, Áreas Protegidas y Vida Silvestre (ICF), que es el ente que se encarga del manejo de las áreas protegidas en Honduras (ICF/DAP).
La RBU protege una biodiversidad variada de flora y fauna, pero sobre todo provee bienes $\mathrm{y}$ servicios ecosistémicos de gran valor. De todos estos bienes y servicios, sin duda el más tangible y apreciado es el agua. Zamorano y varias comunidades aledañas al área protegida en los municipios de San Antonio de Oriente y Tatumbla se benefician de este elemento imprescindible. El área núcleo de la reserva mide solamente 237 ha, sin embargo, es esencial en la regulación del recurso hídrico de toda la montaña y por ende para los usuarios en su pie de monte en el Valle del Yeguare así como otras comunidades del municipio de San Antonio de Oriente y el municipio de Tatumbla.

La RBU, de acuerdo a su categoría de manejo, es de gran importancia debido a su función como regulador de los flujos hídricos, mismos que son favorecidos mediante la captura adicional de agua, producto de la precipitación horizontal. A la vez el 
bosque intercepta y transfiere en forma segura a la superficie del suelo la lluvia proveniente de la precipitación lo que evita la erosión de sus frágiles suelos. Dicha agua continúa su flujo a través del suelo y subsuelo para alimentar y recargar los acuíferos que mantienen estables los caudales en los manantiales y los ríos que se originan en la reserva y fuera de ella. Es por ello que Uyuca juega un papel esencial como proveedor de agua a las comunidades aledañas. Esta función es primordial dado que la mayoría de los bosques de la zona están fuertemente degradados y perdieron este papel, lo que conduce a una mayor presión y dependencia de la RBU. La pérdida de cobertura boscosa, además de afectar los flujos hídricos, afecta a la biodiversidad vegetal que es el sustrato base de la biodiversidad animal. Así se han afectado varias especies de fauna incluidas especies con distribución restringida, endémicas de Honduras, como es el caso de la musaraña Cryptotis hondurensis.

Pese a la constante presión humana a la que se encuentran sometidas las áreas naturales protegidas en Honduras, la RBU constituye un refugio que alberga un importante número de especies vegetales $\mathrm{y}$ animales. Además, la RBU provee servicios ecosistémicos que Zamorano reconoce y valora, por lo cual anualmente invierte en su protección. De esta manera, Uyuca se administra como un recurso invaluable y estratégico para que Zamorano pueda cumplir sus quehaceres institucionales y llevar a cabo en forma efectiva su misión formadora de capital humano para la protección y manejo de las riquezas naturales de toda la región.

\section{Entorno, Historia y Declaratoria de la Reserva Biológica Uyuca (RBU)}

La Reserva Biológica Uyuca está situada a $15 \mathrm{~km}$ al sureste de la ciudad de Tegucigalpa y a $14 \mathrm{~km}$ de la Escuela Agrícola Panamericana (EAP) en el Departamento de Francisco Morazán. La RBU consta de dos zonas delimitadas separadamente en su creación pero necesariamente juntas para su ecología y manejo. Estas dos zonas son la reserva biológica y la zona forestal protegida. Para su manejo, la reserva biológica es la zona núcleo del área protegida y la zona forestal protegida es su área de amortiguamiento (ICF-EAP 2012). La zona núcleo se extiende sobre 237 ha de la parte alta del Cerro Uyuca y fue declarada y delimitada como reserva biológica en 1985. Cuarenta y dos por ciento de la zona núcleo de la RBU pertenece a la EAP. Por Decreto, a partir de 1986, la administración y el manejo técnico de dicha zona de la RBU están a cargo de la EAP (COHDEFOR 1986). Debido a que el nuevo plan de manejo de la RBU aun no está oficializado, se tratará a la zona núcleo como equivalente a la RBU. No obstante, se debe tener en mente que la zona forestal protegida del Cerro Uyuca es y se maneja como la zona de amortiguamiento de la reserva biológica (ICF-EAP 2012).

Mediante los acuerdos ejecutivos números 1348 del 10 de octubre de 1984 y 1853 del 23 de septiembre de 1985, se acordó declarar la Zona Forestal Protegida del Cerro Uyuca con una extensión superficial de 904 ha (COHDEFOR 1986). Estos acuerdos clasificaron como reserva biológica al área superior del Cerro Uyuca comprendida entre la cota de $1,700 \mathrm{msnm}$ y el punto más alto del cerro para una extensión de 237 ha (COHDEFOR 1986). En el convenio de cooperación técnica de 1986 entre COHDEFOR y la EAP se señala que desde su fundación en 1941, Zamorano ha protegido y conservado no solo su propiedad de bosque nublado mixto latifoliado del Cerro Uyuca, sino también las laderas aledañas a este bosque (COHDEFOR 1986). En este documento también se señala que la EAP tomó la iniciativa para la creación de la Reserva Biológica del Cerro Uyuca y colocó parte de su propiedad dentro de esta reserva con el objetivo de realizar estudios ecológicos y técnicos que serían de gran beneficio para la conservación del medio ambiente de Honduras (COHDEFOR 1986).

Ya en 1948, el Dr. Wilson Popenoe, primer director de la EAP, escribió acerca de varias propiedades compradas por la EAP que incluía una del bosque nuboso en Uyuca que sería mantenida como reserva biológica (Monthly News Letter 1948). En este mismo escrito, el Dr. Popenoe señaló que el bosque nuboso es raro en Honduras y que si la EAP no aseguraba esa propiedad, con toda probabilidad sería destruida en el futuro cercano (Monthly News Letter 1948). La mayoría de los bosques que cubrían a otros cerros, así como otras áreas boscosas de la zona del Valle del Yeguare y sus alrededores, han sido convertidos a otros usos, particularmente urbano, ganadería y agricultura. 
El 8 de enero de 1986 se publicó en el Diario Oficial La Gaceta el Decreto Número 211-85, mediante el cual se creó la Zona Forestal Protegida del Cerro Uyuca que incluía a la Reserva Biológica Uyuca (La Gaceta 1986). Con este decreto se protege legalmente al cerro Uyuca como una de las áreas silvestres más importantes de la zona, no solo por su biodiversidad y su exuberancia boscosa, sino por la diversidad de bienes y servicios ecosistémicos que brinda a las comunidades. Estos servicios incluyen el abastecimiento de agua, la regulación del microclima, la captura de carbono, la belleza escénica y otros servicios ecosistémicos que son fundamentales para el sistema de vida de la población humana.

La RBU ha sido establecida legalmente para limitar el alcance de la conversión de tierras dentro de su área delimitada. Sin embargo, el establecimiento de estos límites no es suficiente para garantizar su preservación. La expansión de las actividades agrícolas y no agrícolas, el crecimiento demográfico y el proceso de la urbanización en los municipios de Tatumbla y San Antonio de Oriente, no siempre respetan los límites de la RBU o dependen para su desarrollo de los recursos que provee. Durante la última década, se ha dado un proceso constante de urbanización en ambos municipios. La migración rural-urbana hacia Tegucigalpa, el crecimiento demográfico, el mejoramiento de la carretera Tegucigalpa-Danlí, la construcción ilegal de asentamientos y el desarrollo de nuevas urbanizaciones para la clase media en las zonas periurbanas son algunos factores determinantes que han contribuido a este proceso (Ferrera y Sanders s.f.). Las condiciones urbanas en la región se caracterizan por la presencia de dos municipios medianos de diferente tamaño y densidad poblacional (Tatumbla 62 habitantes por kilómetro cuadrado y San Antonio de Oriente 60 habitantes por kilómetro cuadrado), en los cuales se observa una creciente urbanización no planificada y conflictos por la tenencia de la tierra con respecto al uso del suelo urbano, ganadero y agrícola. Además, existen varias aldeas que dependen de los recursos naturales en diferentes grados (Ferrera y Sanders s.f.).

Desde el punto de vista de conservación biológica, en la región de la RBU se observa una modificación del entorno por los procesos de deforestación que han afectado a las áreas de bosque no protegidas, lo que representa un impacto social y económico a los pobladores que dependen de este recurso como fuente energética (sobre todo los estratos de pobres). Como consecuencia de la deforestación en las áreas no protegidas, hay un aumento de corta ilegal para leña en la RBU (Ferrera y Sanders s.f.).

Las necesidades de agua para consumo humano dentro de los dos municipios se estiman en más de $730,000 \mathrm{~m}^{3}$ al año que son utilizadas por una población de casi 20,000 habitantes, de los cuales $75 \%$ reside en San Antonio de Oriente y el 25\% restante en Tatumbla. Esta demanda de agua para consumo humano se satisface con aguas superficiales (Tatumbla) y subterráneas (sobre todo en la zona del Valle del Yeguare). En total hay 10 aldeas que se benefician directamente por el agua de la RBU. Sin embargo, la presión sobre la RBU es alta. Varias aldeas, tales como el Pedregal y Hoya Grande, están gestionando un mayor acceso a esta fuente y hay planes de las aldeas más lejanas para construir líneas de conducción desde la RBU (Ferrera y Sanders s.f.).

Debido a la historia de su creación, la Reserva Biológica Uyuca tiene una delimitación muy particular. Su decreto de creación establece que la reserva está ubicada desde la cota de 1,700 msnm hasta la parte más alta del Cerro del Uyuca. Este mismo decreto crea la Zona Forestal Protegida (ZFP) desde los 1,700 msnm hasta sus límites inferiores a los 1,300 msnm con 580 ha (La Gaceta 1986). Esta zona actúa como zona de amortiguamiento de la RBU, pero no ha sido formalmente parte de la RBU. La ZFP ha sido un área con metas y objetivos de manejo diferentes e independientes a los de la RBU. Sin embargo, es conveniente la administración conjunta de ambas áreas protegidas como una reserva biológica con su respectiva zona de amortiguamiento (ICF-EAP 2012).

\section{Descripción Biofísica de la Reserva Biológica Uyuca}

Según la clasificación de zonas de vida del sistema de Holdridge (Holdridge 1967), en el área de la RBU existen tres zonas de vida. La RBU se 
encuentra dentro de la zona de vida Bosque muy húmedo montano bajo subtropical (bmh-MBS), la cual está rodeada por el Bosque húmedo montano bajo subtropical (bh-MBS) que es el predominante en la ZFP. Además, en los límites de la ZFP existe una porción del Bosque húmedo subtropical (bh-S).

En el Cerro Uyuca predominan las áreas de laderas con pendientes moderadas a fuertes. Esta característica, aunado a la precipitación de la zona, la temperatura y la duración de la época lluviosa condicionan el tipo de vegetación en la RBU. En la RBU se pueden diferenciar tres tipos de comunidades vegetales, el bosque latifoliado maduro, los bosques mixtos y los relativamente puros dominados por pinabete, además de bosques secundarios en diferentes fases de sucesión. En el bosque latifoliado de Uyuca existe una gran variedad y riqueza de especies de bromelias y epífitas que constituyen hábitats de importancia para otras especies de flora y fauna (ICFEAP 2012). En general, en la RBU se han enlistado 562 especies distribuidas en 113 familias (Pfeifer 1960), aunque este listado está en revisión. Del total de especies reportadas, se encuentran ocho especies endémicas de Honduras, una de Mesoamérica y cuatro en la lista roja de la UICN (Cuadro 3).

Cuadro 3. Especies de plantas de la lista recopilada para la Reserva Biológica Uyuca, Honduras, bajo alguna categoría de conservación.

\begin{tabular}{|c|c|c|c|c|}
\hline \multirow{2}{*}{ Nombre científico } & \multirow{2}{*}{ Hábito } & \multicolumn{3}{|c|}{ Categoría } \\
\hline & & Endémica Honduras & Mesoendémica & UICN \\
\hline Ilex williamsii & Árbol & Sí & & En peligro crítico \\
\hline Schistocarpha longiligula & Arbusto & & Sí & \\
\hline Pentacalia magistri & Arbusto & Sí & & \\
\hline Quercus bumelioides & Árbol & & & Vulnerable \\
\hline Salvia dorisiana & Hierba & Sí & & \\
\hline Salvia kellermanii & Hierba & Sí & & \\
\hline Parathesis vulgata & Árbol & & & En peligro \\
\hline Pinus tecunumanii & Árbol & & & Vulnerable \\
\hline Rubus breviglandifer & Arbusto & Sí & & \\
\hline Rubus hondurensis & Arbusto & Sí & & \\
\hline Rubus shankii & Arbusto & Sí & & \\
\hline Lycianthes hortulana & Arbusto & Sí & & \\
\hline
\end{tabular}

El bosque latifoliado maduro en la RBU cubre 52 ha y tiene entre 30 y 33 especies por hectárea para el conjunto de árboles con diámetro a la altura del pecho (DAP) mayor o igual a $5.0 \mathrm{~cm}$ (Aguilar 2002). Esta diversidad de especies se encuentra distribuida en 19 familias y 27 géneros. El bosque es relativamente complejo con un cociente de mezcla $1 / 6$, lo que indica que en promedio debe aparecer una nueva especie cada seis individuos. La familia Fagaceae es la de mayor peso ecológico dentro de la masa madura, seguida por Lauraceae. A nivel de especies, Persea americana var nubigena ocupa la primera posición en la escala de valor de importancia (IVI) o peso ecológico. La segunda especie en importancia ecológica es Quercus benthamii. Aproximadamente 50\% de la estructura de la comunidad florística está determinada por cinco especies: $P$. americana var nubigena, $Q$. benthamii, Ardisia venosa, $Q$. bumelioides y Miconia theaezans (Aguilar 2002). Cuatro estratos o pisos caracterizan el perfil vertical del bosque maduro: estrato inferior, integrado por individuos con altura total menor a 4.0 $\mathrm{m}$; estrato medio, con árboles entre 4 y $16 \mathrm{~m}$ de altura; estrato superior, con individuos entre 16 y $38 \mathrm{~m}$; estrato emergente, con árboles superiores a los $38 \mathrm{~m}$ de altura (Aguilar 2002).

Los bosques dominados por el pinabete (Pinus 
maximinoi) abarcan unas 107 ha, equivalentes a $45 \%$ de la superficie de la reserva. Este tipo de bosque incluye áreas regeneradas después del ataque de los gorgojos descortezadores del pino y rodales puros o relativamente puros y densos, principalmente asociados con árboles de la familia Fagaceae (EAP 2010). Estos rodales están localizados en las laderas a barlovento. En las faldas a sotavento se encuentran rodales ralos de pinabete debido principalmente a la menor precipitación y a la presencia de suelos superficiales rocosos. En algunos lotes densos de pinabete es posible observar todavía individuos viejos, de más de 200 años de edad y más de $40 \mathrm{~m}$ de altura (Muñoz 2002). Como especie nómada de vida relativamente larga, Pinus maximinoi se asocia, además de la familia Fagaceae, con individuos de las familias Fabaceae, Hamamelidaceae, Clethraceae, Clusiaceae, Betulaceae y Myricaceae (Muñoz 2002). En este tipo de bosque, en especial en las laderas a barlovento, debido a las nubes o neblinas y a la alta humedad relativa del aire, los troncos y copas de los árboles tienen bastante epífitas, principalmente bromelias, orquídeas, musgos, líquenes y helechos (Chang 2000).

El tercer bosque en la RBU es el bosque secundario de hoja ancha que está en diferentes etapas de regeneración. En algunos sitios se encuentran pequeños lotes de robles y encinos (Quercus spp.) asociados con guachipilín (Diphysa americana) (García 2002). No obstante, la mayor parte de la masa de segundo crecimiento está conformada por vegetación baja y tupida. Algunas de las especies de esta comunidad vegetal son Quercus spp., palo de agua (Hedyosmum mexicanum), achiotillo (Vismia baccifera), mora (Carpinus tropicalis), álamo (Carpinus laxiflora), mozote (Triumfetta sp.), mano de león (Citharexylum caudatum), capulín (Trema micrantha) y palo de moco (Saurauia selerorum) (Muñoz 2002).

En la RBU existe una comunidad de orquídeas terrestres y epífitas. Las orquídeas epífitas son más variadas y numerosas, si se comparan con las terrestres (Peñaherrera 1995). De igual manera, las orquídeas epífitas a nivel del bosque latifoliado maduro son también variadas y numerosas al establecer comparaciones con los rodales de Pinus maximinoi. En estudios sobre orquídeas epífitas se ha determinado que en el bosque maduro de la RBU existen al menos 43 especies, donde Arpophyllum spicatum y Stelis parvula son las de mayor peso ecológico (Peñaherrera 1995). En el bosque de P. maximinoi se encontraron seis géneros con una especie cada una, con Scaphyglottis fasciculata y Briegeria teretifolia como las especies de mayor importancia ecológica. En el bosque latifoliado y el de pino, la mayoría de las especies de orquídeas fueron encontradas principalmente en las copas de los árboles (Peñaherrera 1995).

La RBU, debido a su tamaño pequeño, no alberga especies de fauna de gran tamaño, no obstante, algunos grupos como el de los insectos y las aves pueden ser abundantes. Adicionalmente, la RBU funciona como área de conexión para un corredor biológico entre las áreas boscosas de la zona. El proyecto PROARCA COSTAS definió el Corredor Biológico Región del Golfo de Fonseca o cuenca del Pacífico (CBM VIII), del cual forma parte la Reserva de Uyuca (PROARCA/AMP 2002). Por lo tanto, la RBU constituye un sitio importante de paso para diferentes especies de fauna, entre los que se encuentran algunos mamíferos de pequeño y mediano tamaño e incluso de gran tamaño, así como para aves. Aunque la fauna de la RBU ha sido relativamente poco estudiada, existen algunos grupos faunísticos importantes.

En la RBU se han encontrado 16 especies de anfibios y 10 de reptiles (7\% de la herpetofauna de Honduras). En la RBU existen representantes de tres órdenes, 12 familias y 18 géneros de anfibios y reptiles (Townsend et al. 2007). El grupo de las ranas es el más representado en Uyuca. De las especies de herpetofauna registradas, seis se encuentran en la lista roja de especies amenazadas de la UICN bajo alguna categoría (Cuadro 4).

Las aves es uno de los grupos taxonómicos más estudiados en la RBU en donde se han observado 168 especies (Juárez y Komar s.f.), aproximadamente 23\% del total de las especies de aves de Honduras. Los parúlidos (Passeriformes: Parulidae) es la familia más representada en términos del número de especies (27). De todas las familias identificadas, nueve están 
representadas por únicamente una especie. En estudios recientes (SalvaNatura 2011, Juárez y Komar s.f.) la especie Chlorospingus ophthalmicus fue la más abundante durante los muestreos de monitoreo de 2010 y 2011. A pesar de que la mayoría de las especies de aves residentes o estacionales registradas en la RBU están dentro de la categoría de Preocupación Menor de la UICN, algunas son de consideración especial (SERNA 2008). Quince especies de aves de la Reserva (Cuadro 5) se encuentran en alguna categoría de amenazadas de las listas de la UICN o los apéndices CITES (SERNA 2008).
Cuadro 4. Especies de herpetofauna detectadas en la Reserva Biológica Uyuca bajo alguna categoría de amenaza según la Unión Internacional para la Conservación de la Naturaleza (IUCN).

\begin{tabular}{ll}
\hline Especie & UICN \\
\hline Plectrohyla guatemalensis & Peligro crítico \\
Ptychohyla hypomykter & Peligro crítico \\
Ptychohyla salvadorensis & En peligro \\
Craugastor emleni & Peligro crítico \\
Craugastor laevissimus & En peligro \\
Bolitoglossa carri & Peligro crítico \\
\hline
\end{tabular}

Cuadro 5. Especies de aves de la Reserva Biológica Uyuca incluidas en las listas de la Unión Internacional para la Conservación de la Naturaleza (IUCN) o la Convención sobre el Comercio Internacional de Especies Amenazadas de Fauna y Flora Silvestres (CITES).

\begin{tabular}{llll}
\hline Especie & Nombre en español & \multicolumn{1}{c}{ UICN } & CITES \\
\hline Accipiter striatus & Gavilán pajarero & Preocupación menor & II \\
Bolborhynchus lineola & Perico rayado & Preocupación menor & II \\
Buteo albonotatus & Aguililla aura & Preocupación menor & II \\
Buteo brachyurus & Gavilán colicorto & Preocupación menor & II \\
Buteo jamaicensis & Águila colirroja & Preocupación menor & II \\
Buteo platypterus & Gavilán ala ancha & Preocupación menor & II \\
Buteogallus anthracinus & Gavilán cangrejo & Preocupación menor & II \\
Elanus leucurus & Milano coliblanco & Preocupación menor & II \\
Ciccaba virgata & Lechuza café & Preocupación menor & II \\
Cyrtonyx ocellatus & Colín ocelado & Vulnerable & \\
Dendroica chrysoparia & Chipe dorsinegro & En peligro & II \\
Falco sparverius & Kli-klis & Preocupación menor & II \\
Micrastur ruficollis & Halcón selvático rayado & Preocupación menor & III \\
Sarcoramphus papa & Rey zope & Preocupación menor & \\
Vermivora chrysoptera & Chipe alidorada & Casi Amenazado & \\
\hline
\end{tabular}

Setenta y seis especies de mamíferos (en 20 familias y nueve órdenes) incluían en su ámbito original de distribución a la RBU. De estos, el orden más numeroso es el de murciélagos (Chiroptera) con 35 especies. Así que, potencialmente cualquiera de estas especies podría aparecer en Uyuca aunque sea de manera esporádica. De estas especies de mamíferos esperadas (existentes o de aparición eventual) en la RBU, presentan algún grado de amenaza: el pizote (Nasua narica), el mico de noche (Potos flavus) y el lepasil (Eira barbara), incluidos en el apéndice III de
CITES para Honduras (SERNA 2008). En la RBU existe la musaraña Cryptotis hondurensis, conocida solo de tres lugares en Honduras que están en el departamento de Francisco Morazán, lo cual significa que la especie es endémica de esta zona del país (Woodman y Timm 1992). Algunos mamíferos comunes son: el ratón de patas blancas (Peromyscus mexicanus), la comadreja (Mustela frenata) y el guazalo (Didelphis marsupialis).

En la RBU se ha estudiado la fauna de macroinvertebrados acuáticos que son un componente 
importante de la biodiversidad en los sistemas lóticos. Estos organismos son importantes desde el punto de vista ecológico porque constituyen la base de la red alimentaria ya que sirven de alimento a organismos de mayor tamaño tales como los peces, ranas y aves (Roldán 1992). Además, las comunidades de macroinvertebrados acuáticos tienden a variar si las condiciones de los ríos cambian, de aquí que su estudio sea importante (Lampert y Sommer 2007). En la RBU se muestrearon dos sitios, uno en la quebrada La Pita y otro en la quebrada Agua Amarilla. La composición de la comunidad de macroinvertebrados fue más alta en la quebrada Agua Amarilla, con más individuos, pero con menos taxa que en la quebrada $\mathrm{La}$ Pita (López 2008). La comunidad de estos sitios estuvo compuesta por 984 individuos de 59 taxa. El orden Hemiptera fue el más abundante con $33 \%$ de los individuos. Entre las especies sobresalen miembros de Gomphidae, Leptophlebiidae y Blaberidae que solo se encuentran donde el agua está limpia (Roldán 2003).

\section{Manejo de la Reserva Biológica Uyuca}

En la RBU y en toda la región en general existe una problemática ambiental y socioeconómica relacionada con las actividades humanas producto del cambio de uso del suelo. La expansión de la agricultura y la extracción de leña y otros productos del bosque tales como musgos y bromelias, constituyen los principales problemas de la RBU y la ZFP. Este tipo de problemas en la RBU provocan efectos negativos como pérdida de hábitats, especies y la pérdida del suelo producto de la erosión, así como una disminución eventual del recurso hídrico (Ferrera y Sanders s.f.). Estas razones fueron las impulsoras de la creación de la RBU. Pero la creación no basta para cumplir con los objetivos y metas propuestas para la RBU. Por lo tanto, se requieren acciones de manejo de áreas protegidas acorde con la categoría de manejo de la RBU para garantizar su persistencia a largo plazo a pesar de la eterna amenaza externa que experimentan las áreas protegidas (Janzen 1986).

La RBU forma parte del Sistema Nacional de Áreas Protegidas de Honduras (SINAPH) a cargo del
ICF, es una de las 91 áreas protegidas (entre declaradas y propuestas) de Honduras (Vreugdenhil et al. 2002). Debido a ello, en 1988 Zamorano presentó el primer Plan de Manejo de la ZFP que incluyó a la RBU. Este plan de manejo fue parte de los compromisos adquiridos por Zamorano al firmar el convenio de comanejo con el ICF (COHDEFOR en ese momento), pero a la vez constituye un elemento más que ha demostrado el compromiso ambiental de Zamorano. En ese plan se contempló una combinación de metodologías para la conservación de los recursos naturales de todo el Cerro Uyuca (Agudelo 1986).

Zamorano trabaja en varios programas para la protección de la zona forestal protegida, entre los que se encuentran la reforestación de más de 20 ha de bosque afectadas por el gorgojo, la vigilancia y el combate de incendios, el monitoreo de plagas y la rehabilitación de más de $46 \mathrm{~km}$ de rondas contra incendios (EAP 2010). Durante 2011 y 2012, Zamorano elaboró un nuevo plan de manejo exclusivo para la RBU e incluye a la ZFP como la zona de amortiguamiento. Se elaboró con los lineamientos establecidos por el ICF (ICF-EAP 2012) y con participación comunitaria. El plan fue elaborado con los recursos técnicos y científicos de Zamorano y el apoyo de varios actores que incluyó a la Municipalidad de San Antonio de Oriente, la Municipalidad de Tatumbla, Las Fuerzas Armadas de Honduras y el ICF. Con este plan se cuenta con las estrategias y recursos financieros necesarios para el cumplimiento de los programas de manejo y administración que permitan la conservación de la biodiversidad y otros bienes y servicios ecosistémicos que la RBU provee.

Entre los objetivos y alcances del plan de manejo está el manejo que permita conservar la salud de los ecosistemas del sitio y velar por el cumplimiento de las actividades que se deben implementar. Las estrategias de conservación permitirán la reducción de las amenazas críticas actuales y potenciales sobre los bienes y servicios ecosistémicos que provee. Se espera, no solo mejorar la viabilidad de los objetos de conservación dentro del área, sino lograr la conservación a largo plazo de los bienes, los procesos y los servicios imprescindibles para las comunidades. 


\section{Plan de Manejo de la Reserva Biológica Uyuca}

Desde antes de su declaratoria, Zamorano ha protegido e investigado los diferentes componentes de la RBU. Toda la información generada y el plan de manejo implementado desde 1988 sirvieron de insumos para la elaboración del plan de manejo actual. Este fue elaborado por un equipo interdisciplinario de la Zamorano que a la vez es responsable de la ejecución del plan. El plan contiene cuatro programas y varios subprogramas de manejo con un costo mínimo de \$400,000 para los cinco años de su implementación.

El plan de manejo de la RBU ha sido establecido para 5 años. El objetivo general del plan es proteger, preservar y recuperar los ecosistemas de la RBU para asegurar la estabilidad del recurso hídrico así como otros bienes y servicios ecosistémicos, implementando políticas de conservación a perpetuidad y acciones de protección y conservación del medio natural. Como objetivos específicos se ha establecido el asegurar la protección y conservación de la biodiversidad e integridad ecológica de la RBU; mejorar y consolidar la capacidad de manejo de la administración de la RBU; incorporar la política de conservación y manejo de áreas protegidas en las políticas sectoriales y regionales e incrementar el conocimiento científicotécnico interdisciplinario aplicado al manejo de los ecosistemas y biodiversidad de la RBU (ICF-EAP 2012).

Los programas y subprogramas incluidos son el Programa de Manejo Integrado de Recursos Naturales con los subprogramas de Protección Forestal y Manejo de Microcuencas; el programa de Administración con los subprogramas de Gestión y Comanejo, de Infraestructura y de Personal; el programa de Educación Ambiental; y el programa de Investigación que incluye también las actividades correspondientes de monitoreo. El plan también incluye una Estrategia de Implementación del Plan con su cronograma correspondiente y su respectivo presupuesto.

\section{Visión, Misión y Objetivos}

Visión. La RBU y su área de influencia son áreas de conservación y preservación de recursos. En la RBU están inmersas las zonas de producción hídrica con altos niveles de calidad y cantidad de este recurso, primordialmente para los habitantes de la región de El Zamorano y sus poblaciones rurales y en segunda instancia para los sistemas productivos que así lo requieran (ICF-EAP 2012).

Misión. Conservar, proteger y administrar la RBU y su área de influencia como fuentes abastecedoras del recurso hídrico y demás bienes y servicios ecosistémicos, así como la biodiversidad de la RBU a largo plazo mediante acciones de conservación, protección y recuperación de las áreas que han sido alteradas (ICF-EAP 2012).

Objetivos. El artículo 324-5 del Reglamento General de la Ley Forestal, Áreas Protegidas y Vida Silvestre define a la reserva biológica como: "Área que, por los ecosistemas, rasgos o flora y fauna de alto valor científico que contiene, es inexplotable y forma parte del patrimonio nacional. Su función principal es proteger, conservar y mantener fenómenos o procesos naturales en estado inalterado, para estudios $\mathrm{e}$ investigación científica bajo estricto control de las autoridades competentes". Es por ello que el objetivo general de este plan de manejo está acorde con esta definición (ICF-EAP 2012).

\section{Zonificación}

Uno de los aspectos principales y fundamentales para el manejo de un área protegida es la zonificación. Debido al decreto de creación, la RBU es la Zona de Preservación Absoluta o Zona núcleo. La ZFP (creada mediante el mismo decreto) es la zona de amortiguamiento de la RBU. Para mayor claridad, a la hora de ejecutar las actividades se optó por dividir la RBU en subzonas para manejo (Figura 1). Se establecieron cuatro subzonas: la subzona de uso especial, la subzona de uso restringido, la subzona de protección absoluta y la subzona de Restauración Ecológica. La meta es garantizar la viabilidad de los objetos de conservación establecidos en la RBU por medio de la implementación del plan de manejo mediante las diferentes actividades a desarrollar según los sectores identificados dentro de cada subzona (ICFEAP 2012). 


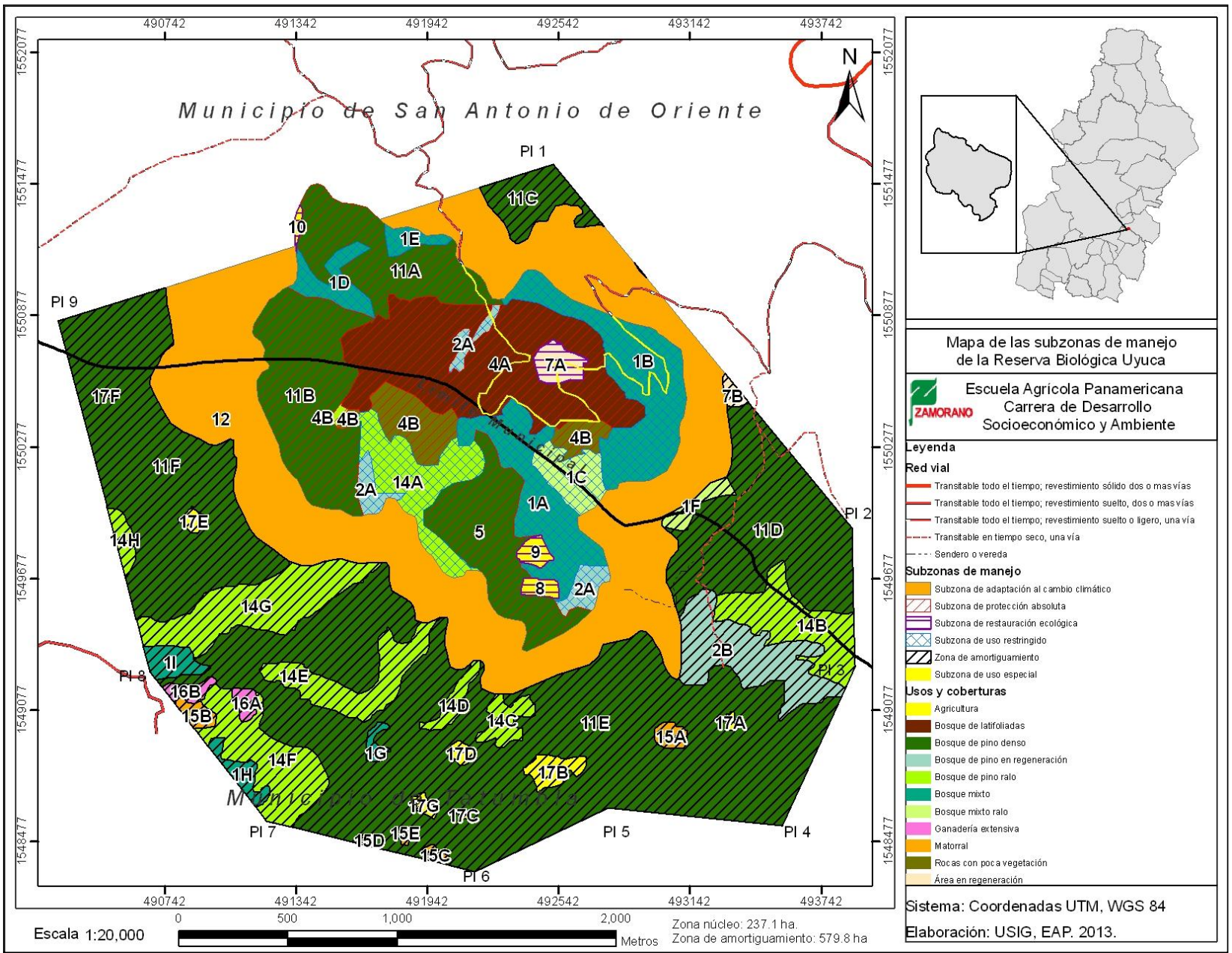

Figura 1. Subzonas de manejo por sectores (números) de la Zona Núcleo de la Reserva Biológica Uyuca, Francisco Morazán, Honduras.

\section{El Concepto de Comanejo y el Manejo Actual de la Reserva Biológica Uyuca}

Un aspecto primordial para el manejo actual de las áreas protegidas es el tipo de gobernabilidad que las rige. Existen al menos cuatro tipos de gobernabilidad en donde la más familiar es aquel en que las áreas protegidas son manejadas por el gobierno de un estado o país; otras gobernabilidades son el comanejo, la privada y la conservada por la comunidad (Cuadro 6).
Cuadro 6. Cuatro tipos de gobernabilidad que se dan en las áreas protegidas.

\begin{tabular}{lll}
\hline Gobernabilidad & Encargado & Estado \\
\hline Gobierno & $\begin{array}{l}\text { Ministerio o } \\
\text { agencia estatal } \\
\text { Múltiples }\end{array}$ & Tradicional \\
Comanejo & $\begin{array}{l}\text { actores } \\
\text { Propietarios } \\
\text { privados }\end{array}$ & $\begin{array}{l}\text { Larga } \\
\text { historia }\end{array}$ \\
Privada & $\begin{array}{l}\text { Fomunidades } \\
\text { indígenas }\end{array}$ & $\begin{array}{l}\text { Forma más } \\
\text { antigua }\end{array}$ \\
\hline
\end{tabular}


El comanejo va en aumento como respuesta a los derechos de la sociedad. Se usan procesos complejos y mecanismos institucionales para compartir la responsabilidad y la autoridad del manejo entre el gobierno y la sociedad civil. En el manejo colaborativo, la responsabilidad del manejo y la toma de decisiones pueden estar en manos de una agencia, comúnmente del gobierno. No obstante, la agencia debe colaborar, por ley o por política, con los otros interesados con informes o consulta a sus socios, hasta los casos donde los actores desarrollen y aprueben las regulaciones y las propuestas de manejo (BorriniFeyerabend et al. 2004). Las iniciativas privadas tienen tradición y brindan un gran apoyo a los gobiernos locales y estatales en su lucha por la conservación, ya que tienen limitaciones para la protección in situ de la biodiversidad (Mora 2002). Por último, están las reservas que han sido conservadas por las comunidades indígenas. Esta podría ser la forma más antigua de gobernabilidad y sigue siendo una forma común de manejo (Borrini-Feyerabend et al. 2004).

Las áreas protegidas cubren unos 20 millones de kilómetros cuadrados, casi $12 \%$ de la superficie terrestre del planeta, pero solo $3.5 \%$ de la superficie total, ya que existen pocas áreas marinas protegidas (Chape et al. 2003). Hay áreas protegidas en casi todos los países y son manejadas con diferentes esquemas, generalmente para la conservación de la biodiversidad. No obstante, esta tendencia ha cambiado ya que los sistemas de áreas protegidas han tenido que responder a demandas sociales y económicas de la sociedad. Por eso, cada vez más se acepta la necesidad de armonizar el desarrollo de las áreas protegidas con las necesidades sociales y de desarrollo económico local, tal y como se estableció en la convención de diversidad biológica en la conferencia de ambiente y desarrollo de Río en 1992 (ONU 1992). En este sentido, la percepción de un área protegida cambió de un entendimiento convencional de áreas aisladas establecidas tecnocráticamente y manejadas por los gobiernos, a un entendimiento emergente de áreas protegidas como parte de un sistema mayor con la participación de varios actores en su manejo y objetivos (Cuadro 7).

Las áreas protegidas fueron establecidas con fines de conservación y en algunos casos entretenimiento. Esas primeras áreas protegidas fueron creadas para proteger ambientes frágiles, paisajes excepcionales, vida silvestre o lugares para recreo. Dicho enfoque genera conflictos con comunidades que han sido severamente limitadas en términos del uso de los recursos naturales que esas áreas encierran o incluso han sido expulsados de sus tierras muchas veces sin ninguna compensación.

La Dirección de áreas protegidas del ICF señala que el comanejo en las áreas protegidas en Honduras ha sido implementado desde 1997 a través del ICF. Desde entonces, se han establecido 33 convenios de comanejo en 43 áreas protegidas para una superficie de $2,144,850$ ha. No obstante, el convenio de comanejo de la RBU data de 1986, 11 años antes de la puesta en marcha oficial de esta modalidad de manejo. En este sentido, Zamorano fue pionero en el sistema de comanejo de áreas protegidas en Honduras, labor que continua con los entes correspondientes por ley. El plan de manejo incluye la participación comunitaria y se ha formado un grupo de apoyo (GARBU) para el cumplimiento del plan con representantes de varios sectores de interés incluidas las municipalidades de Tatumbla y San Antonio de Oriente.

Para medir el cumplimiento de sus metas, el ICF evalúa la Efectividad de Manejo de las áreas protegidas del país, mediante una herramienta de 35 indicadores en cinco ámbitos: social, administrativo, recursos naturales y culturales, político-legal y económico-financiero. La Efectividad de Manejo de la RBU fue evaluada en 2010-2012. En el 2011, la RBU obtuvo 845 puntos y en 2012 obtuvo 923 puntos lo que la hace el área protegida de Honduras con mayor calificación. Solo cuatro áreas protegidas de Honduras han obtenido puntajes sobre los 800 puntos: Parque Nacional La Tigra, Jardín Botánico Lancetilla, Parque Nacional Cerro Azul Meambar y ahora la RBU ${ }^{3}$. Así Zamorano demuestra su capacidad para manejar un área protegida de alta fragilidad. Para Zamorano es halagador que la RBU esté en el nivel de manejo satisfactorio, ya que ha sido un gran esfuerzo y ha invertido en recursos económicos y de personal para lograrlo. El compromiso de Zamorano es seguir manteniendo el nivel satisfactorio en la efectividad del manejo, pero sobre todo contribuir a la conservación ambiental y la biodiversidad de Honduras.

\footnotetext{
${ }^{3}$ Marlenia Acosta, ICF., comunicación personal.
} 
Cuadro 7. El nuevo paradigma para las áreas protegidas en relación a su antigua percepción de manejo (Adaptado de Phillips 2002).

\begin{tabular}{|c|c|c|}
\hline Tópico & Percepción convencional & Percepción actual \\
\hline Objetivos & $\begin{array}{ll}\text { - } & \text { Creadas para conservación } \\
\text { - } & \text { Protección escénica y fauna } \\
& \text { silvestre } \\
\text { - } & \text { Manejada para visitantes y turistas } \\
\text { - } & \text { Valoradas como áreas silvestres } \\
\text { - } & \text { Protección }\end{array}$ & $\begin{array}{l}\text { - } \text { Con objetivos sociales y económicos } \\
\text { - } \text { A veces creadas por razones científicas, } \\
\text { económicas y culturales } \\
\text { - } \text { Manejada con la gente local en mente } \\
\text { - } \text { Importancia cultural de la naturaleza } \\
\text { - }\end{array}$ \\
\hline Gobernabilidad & Gobierno central & Variedad de actores e interesados \\
\hline $\begin{array}{l}\text { Comunidades } \\
\text { locales }\end{array}$ & $\begin{array}{l}\text { Planeadas y manejadas en contra } \\
\text { de la gente } \\
\text { - Manejadas sin importar la opinión } \\
\text { local }\end{array}$ & $\begin{array}{l}\text { - Manejadas con, para y, en algunos casos, por la } \\
\text { gente local } \\
\text { - Manejadas para llenar las necesidades de la } \\
\text { gente local }\end{array}$ \\
\hline Contexto amplio & $\begin{array}{l}\text { - } \text { Desarrolladas separadamente } \\
\text { - Manejadas como islas }\end{array}$ & $\begin{array}{l}\text { - Planificadas como parte de sistemas nacionales, } \\
\text { regionales e internacionales } \\
\text { - Desarrolladas como redes que incluyen áreas } \\
\text { protegidas, zonas de amortiguamiento y } \\
\text { corredores biológicos }\end{array}$ \\
\hline Percepciones & $\begin{array}{ll}\text { - } & \text { Vistas como bienes nacionales } \\
\text { - } & \text { Vistas solamente como de interés } \\
\text { nacional }\end{array}$ & $\begin{array}{ll} & \text { Vistas también como bienes de la comunidad } \\
\text { - } & \text { Vistas también como de interés internacional }\end{array}$ \\
\hline $\begin{array}{l}\text { Técnicas de } \\
\text { manejo }\end{array}$ & $\begin{array}{ll}\text { - } & \text { Manejo a corto plazo } \\
\text { - } & \text { Manejo de manera tecnocrática }\end{array}$ & $\begin{array}{ll}\text { - } & \text { Manejo adaptativo a largo plazo } \\
\text { - } & \text { Manejo con consideraciones políticas }\end{array}$ \\
\hline Financiamiento & Impuestos & Variedad de fuentes \\
\hline $\begin{array}{l}\text { Habilidades de } \\
\text { manejo }\end{array}$ & $\begin{array}{ll}- & \text { Manejado por científicos y } \\
\text { expertos en recursos naturales } \\
\text { - } & \text { Guiado por expertos }\end{array}$ & $\begin{array}{ll}\text { - } & \text { Manejados por individuos de habilidades } \\
\text { múltiples } \\
\text { - } \quad \text { Manejados con conocimiento local }\end{array}$ \\
\hline
\end{tabular}

\section{Conclusiones}

Las áreas protegidas proporcionan beneficios concretos y potenciales para la humanidad y su desarrollo económico y bienestar. Es por ello, que la creación, filosofía y manejo deben adaptarse a los cambios de las circunstancias en que estas existen. El desarrollo del sistema de áreas protegidas debe ser integrado y parte del progreso económico. Se deben impulsar las actividades no extractivas, en donde el ecoturismo ha mostrado ser una de las más efectivas.

Los estudios de capacidad de carga turística son importantes en la gestión de las áreas protegidas. Pero hay aspectos de mayor prioridad en los cuales trabajar, como la erradicación de la extracción ilegal de productos que causa más daño que el turismo intensivo. La prospección farmacéutica y el pago de servicios ambientales por las áreas protegidas deben ser apoyados y estimulados. Las áreas protegidas deben fortalecer la conservación de la biodiversidad dentro y fuera de ellas mediante sistemas integrados de áreas protegidas, zonas de amortiguamiento $\mathrm{y}$ corredores biológicos.

La integración y el papel de las comunidades locales son fundamentales para su desarrollo y conservación. Los gobiernos centrales y locales deben propiciar el comanejo y apoyar las actividades de buen manejo, incluidas la investigación científica que permita respaldar la sostenibilidad del desarrollo aunado a la preservación de la biodiversidad. 
La RBU es esencial para la provisión de agua a los municipios de Tatumbla y San Antonio de Oriente. Además, provee otros bienes y servicios ecosistémicos menos tangibles, pero importantes para sus habitantes. Se debe educar a las comunidades locales sobre estos beneficios y la necesidad de proteger la RBU.

Durante más de 60 años, Zamorano ha manejado los bosques del Cerro Uyuca con un enfoque cada vez más hacia la preservación de la RBU y su biodiversidad para asegurar la persistencia de los bienes y servicios ecosistémicos que provee. El nuevo plan de manejo ha sido elaborado con participación de Zamorano, del ICF y de otros interesados como la municipalidad de Tatumbla y San Antonio de Oriente.

Gracias a los principios establecidos desde 1948 por el Dr. Wilson Popenoe y una visión cada vez más en pro de los aspectos ambientales, Zamorano ha logrado la conservación de la RBU. Este logro se manifiesta por la conservación de la biodiversidad así como en la posibilidad de contar con los servicios ecosistémicos que provee. De éstos, el más tangible y esencial es el agua que provee a cientos de usuarios en los municipios de San Antonio de Oriente y Tatumbla.

Pese a la constante presión a la que se encuentran sometidas las áreas protegidas de Honduras por las intervenciones humanas, la RBU alberga muchas especies vegetales y animales. Además, provee servicios ecosistémicos que Zamorano reconoce e invierte en su protección. De esta manera, Uyuca se administra como un recurso invaluable y estratégico para que Zamorano pueda cumplir su misión formadora de capital humano para la protección y manejo de las riquezas naturales de toda la región. Con acciones y actividades tales como proyectos especiales de graduación en áreas como la conservación biológica y el manejo de bosques, Zamorano, por medio de la RBU, ha contribuido en la formación de un alto número de profesionales de toda Latinoamérica.

\section{Literatura Citada}

Agudelo, N. 1986. Plan de manejo para el bosque del Uyuca de la Escuela Agrícola Panamericana, El Zamorano, Honduras, primeros cinco años. Tesis Magister Scientiae. Centro Agronómico Tropical de Investigación y Enseñanza. Turrialba, Costa Rica. 327 p.
Aguilar, S.I. 2002. Estudio florístico-estructural de una asociación vegetal en el bosque latifoliado maduro de la Montaña de El Uyuca. Tesis Licenciatura. Escuela Agrícola Panamericana, Zamorano. Honduras. 72 p.

Arguedas M., B. Castaño y J.M. Rodríguez (eds.). 2004. Lineamientos y herramientas para un manejo creativo de las áreas protegidas. Programa de Políticas y Ciencias Ambientales. Organización para Estudios Tropicales. San José, Costa Rica. 729 p.

Beltrán, J. (Ed.). 2001. Pueblos Indígenas y Tradicionales y Áreas Protegidas: Principios, Directrices y Casos de Estudio. UICN, Gland, Suiza y Cambridge, UK y WWF Internacional, Gland, Suiza. 139 p.

Borrini-Feyerabend, G., A. Kothari y G. Oviedo. 2004. Indigenous and local communities and protected areas: Towards Equity and Enhanced Conservation. IUCN, Gland, Suiza y Cambridge, United Kingdom. 111 p.

Chang, P.A. 2000. Guía ilustrada para la identificación de 31 especies de helechos encontradas en la Reserva Biológica de la Montaña Uyuca, Honduras, C.A. Tesis Licenciatura. Escuela Agrícola Panamericana, Zamorano. Honduras. 60 p.

Chape, S., S. Blyth, L. Fish, P. Fox y M. Spalding (Compiladores). 2003. United Nations List of Protected Areas. IUCN, Gland, Suiza y Cambridge, UK y UNEP-WCMC, Cambridge, United Kingdom. $44 \mathrm{p}$.

COHDEFOR (Corporación Hondureña de Desarrollo Forestal). 1986. Convenio de cooperación técnica entre la "Corporación Hondureña de Desarrollo forestal" (COHDEFOR) y la "Escuela Agrícola PanamericanaEAP" (Zamorano). Corporación Hondureña de Desarrollo forestal. Tegucigalpa, Honduras. $6 \mathrm{p}$.

Dasmann, R.F. 1981. Wildlife Biology. Segunda edición. John Willey y Sons. New York. 212 p.

Carey, C., N. Dudley y S. Stolton. 2000. Threats to protected areas. Squandering paradise? The importance and vulnerability of the world's protected areas, World Wide Fund for Nature International, Gland, Switzerland. $226 \mathrm{p}$.

EAP (Escuela Agrícola Panamericana). 2010. Plan de protección forestal 2011. Ms. 12 p.

Ferrera, I. y A. Sanders. s.f. Áreas Naturales y el Desarrollo Rural Equitativo y Sustentable en el Distrito Central. Ms. 19 p.

García, R. 2002. Biología de la Conservación: conceptos y prácticas. Instituto Nacional de Biodiversidad, INBio. Heredia, Costa Rica. 168 p.

Holdridge, L.R. 1967. Life Zone Ecology. Tropical Science Center. San José, Costa Rica. 
ICF (Instituto Nacional de Conservación y Desarrollo Forestal, Áreas Protegidas y Vida Silvestre) y EAP (Escuela Agrícola Panamericana). 2012. Plan de Manejo Reserva Biológica Uyuca. Valle del Zamorano. $128 \mathrm{p}$.

Janzen, D.H. 1986. The eternal external threat. In: M.E. Soulé (ed.). Conservation biology, the science of scarcity and diversity. Sinauer, Sunderland. p. 286303.

Júarez, R. y O. Komar. S.f. Monitoreo permanente de aves en la Reserva Biológica Uyuca, Honduras, 2010-2011. Informe Final. SalvaNATURA, San Salvador, El Salvador. 27 p.

La Gaceta: Diario oficial de la República de Honduras. 1986. Decreto número 211-85. República de Honduras, Tegucigalpa. $10 \mathrm{p}$.

Lampert, W. y U. Sommer. 2007. Limnoecology: The ecology of lakes and streams. 2da edición. Oxford University Street. New York. 324 p.

López, L. 2008. Análisis y valoración de varios índices bióticos mediante la utilización de macroinvertebrados acuáticos en la subcuenca del Yeguare, Honduras. Tesis Magister Scientiae. Escuela de Biología, Universidad de Costa Rica. Costa Rica. 153 p.

Meffe, G.K. y C.R. Carroll. 1997. Principles of Conservation Biology. 2da edición. Sinauer Associates Publishers, Sunderland, Massachusetts. 729 p.

Mora, J. 2002. Plan de manejo y desarrollo del rancho Mastatal. Puriscal, Costa Rica. 80 p.

Muñoz, R. 2002. Estudio florístico-estructural de una asociación vegetal en el bosque latifoliado maduro de la Montaña de El Uyuca. Tesis Licenciatura. Escuela Agrícola Panamerica, Zamorano. Honduras. 77 p.

Myers, N. 1987. The extinction spasm impending: Synergisms at work. Conservation Biology 1(1):1421.

Peñaherrera, C.V. 1995. Inventario sistemático de orquídeas epífitas del bosque nublado del Cerro Uyuca. Tesis Licenciatura. Escuela Agricola Panamerica, Zamorano. Honduras. $86 \mathrm{p}$.

Pfeifer, H.W. 1960. Vascular plants of Mount Uyuca. Ceiba $8(3): 102-141$

Phillips, A. 2002. Directrices de gestión para áreas protegidas de la categoría V de la UICN: paisajes terrestres y marinos protegidos. Comisión Mundial de Áreas Protegidas (CMAP). Serie de directrices sobre buenas prácticas en áreas protegidas No. 9. UICN Unión Mundial para la Naturaleza. 141 p.
Primack, R., R. Rozzi, P. Feinsinger, R. Dirzo y F. Massardo. 2001. Fundamentos de conservación biológica: perspectivas latinoamericanas. Fondo de cultura económica. México. 797 p.

PROARCA/AMP. 2002. Caja de Herramientas para el Manejo de Áreas Protegidas. USAID, CCAD. 16 p.

Redford, K.H. y B. Richter. 2002. Sustainable development and biodiversity preservation are incompatible goals. In: W. Dudley (ed.). Biodiversity. Greenhaven Press, San Diego, California. p. 192-194.

Roldán, G. 1992. Fundamentos de limnología neotropical. Universidad de Antioquia. Medellín, Colombia. 529 p.

Roldán, G. 2003. Bioindicación de la calidad del agua en Colombia: Uso del método BMWP/Col. Universidad de Antioquia. Colombia. 169 p.

SalvaNatura. 2011. Monitoreo Permanente de Aves en Reserva Biológica Monte Uyuca, Honduras, durante 2010: Primer Informe Anual. SalvaNATURA Zamorano, ICF. 19 p.

SERNA (Secretaría de Recursos Naturales y Ambiente). 2008. Especies de Preocupación Especial en Honduras. SERNA/DIBio.Tegucigalpa, Honduras. 77 p.

Townsend, J.H., L.D. Wilson y J.I. Restrepo. 2007. Investigaciones sobre la herpetofauna en el Parque Nacional Montaña de Yoro y la Reserva Biológica Cerro Uyuca, Honduras. Centro Zamorano de Biodiversidad. $11 \mathrm{p}$.

UICN (Unión Mundial para la Naturaleza). 1994. Directrices para las categorías de manejo de áreas protegidas. La comisión de parques nacionales y áreas protegidas de la UICN con la ayuda del Centro Mundial de Monitoreo de la Conservación. 94 p.

ONU (Naciones Unidas). 1992. Convenio sobre la biodiversidad biológica. (en línea). Disponible en: http://www.cbd.int/.

Vreugdenhil, D., P.R. House, C.A. Cerrato, R.A. Martínez y A.C. Pereira. 2002. Rationalisation of the Protected Areas System of Honduras, Volume 1: Main Study. (en línea). Disponible en http://www. birdlist.org/cam/honduras/Rationalisation. pdf.

Woodman, N. y R. M. Timm. 1992. A new species of smalleared shrew, genus Cryptotis (Insectivora: Soricidae), from Honduras. Proc. Biol. Soc. Wash. 105(1): 1-12.

Recibido para publicación el 14 de abril de 2012

Aceptado para publicación el 6 de octubre de 2012. 